\title{
On the Superpropagator of Fields with Exponential Coupling
}

\author{
H. Lehmann and K. Pohlmeyer \\ II. Institut für Theoretische Physik der Universität Hamburg
}

Received July 7, 1970

\begin{abstract}
We define the vacuum expectation value of the time-ordered product of two exponentials of free fields as a distribution using minimal singularity as a criterion. The implication of this definition for an exponentially self-coupled scalar field is studied in second order of a perturbation expansion.
\end{abstract}

\section{Introduction}

Lagrangians which involve an exponential of a spin zero field occur in a number of field-theoretic problems. Usually they result from the application of a formal canonical transformation to an originally polynomial Lagrangian. All exponential interactions are, of course, nonrenormalizable according to the standard classification. However, a number of authors have tried to argue that for exponential couplings the definition of higher order perturbation theory terms need not suffer from the well-known ambiguities of non-renormalizable polynomial models. There is, in particular, an early paper by Okubo [1] and more recent publications by Volkov [2]. The essential idea is to expand only in powers of the interaction Lagrangian, leaving the exponential unexpanded.

In this paper we present a partial analysis of the structure of exponential interactions. For a discussion of the general term in a perturbation expansion it is necessary to give mathematical meaning to the following formal expression:

$$
T: e^{f \phi\left(x_{1}\right)}: \ldots: e^{f \phi\left(x_{n}\right)}:=e^{i f^{2} \sum_{i>j} \Delta_{F}\left(x_{1}-x_{j}\right)}: e^{f \phi\left(x_{1}\right)} \ldots e^{f \phi\left(x_{n}\right)}:
$$

where $\phi$ is a free scalar field of mass $m, f$ a constant. Our present work is restricted to the simplest non-trivial case, the definition of

$$
i E_{F}(12)=\left\langle 0\left|T:\left(e^{f \phi(1)}-1\right)::\left(e^{f \phi(2)}-1\right):\right| 0\right\rangle=e^{l f^{2} \Delta_{F}(12)}-1
$$

which is often called the superpropagator for the exponential interaction. Without further restrictions, Eq. (2) contains an infinite number of arbitrary parameters, due to the singular structure of $\Delta_{F}$. Previous work

8 Commun. math. Phys., Vol. 20 
by Volkov [2] and by Filippov [3] has suggested (at least for $m=0$ ) a definite choice of these parameters. Using a different approach we show that this choice can be characterized as the least singular superpropagator. In a sense to be specified, it corresponds most closely to a given classical Lagrangian.

The existence of a least singular definition of $\exp \left(i f^{2} \Delta_{F}\right)$ distinguishes the exponential interaction from polynomial non-renormalizable Lagrangians since it does not seem possible to give an analogous definition of $\Delta_{F}^{n}$. It should be noted however, that regardless of the choice of a superpropagator all non-polynomial interactions remain extremely singular. In perturbation theory they lead necessarily to cross sections which increase strongly with energy. Whether an analysis of their structure is relevant to problems of physics remains so far, in our opinion, an open question.

In a number of recent papers Efimov [4], Salam and co-workers [5], B. Lee and Zumino [6] and Keck and J. G. Taylor [7] have discussed other non-polynomial interactions. We prefer the exponential interaction for two reasons. Firstly, it belongs to the class of strictly local field theories which has been defined by Jaffe [8]. Secondly, its $S$-matrix elements have a particularly simple structure due to the simple relation between time-ordered and normal-ordered products given by Eq. (1).

\section{Definition of the Superpropagator}

We define in this section a distribution $E_{F}(x)$ which corresponds to the formal relation (2). According to Jaffe [8], the unordered expression

$$
i E^{(+)}(12)=\left\langle 0\left|:\left(e^{f \phi(1)}-1\right):\left(e^{f \phi(2)}-1\right):\right| 0\right\rangle=e^{i f^{2} \Delta^{(+)}(12)}-1
$$

is a well-defined distribution on the space $\mathscr{C}\left(R^{4}\right)$ of test functions. Jaffe shows moreover that $E_{F}(x)$ can also be defined on $\mathscr{C}\left(R^{4}\right)$. However, without further conditions, the definition is not unique. The arbitrariness can be expressed as

$$
\Delta\left(E_{F}(x)\right)=\sum_{n=0}^{\infty} a_{n} \square^{n} \delta^{(4)}(x)
$$

with $a_{n}$ real and $\sum a_{n} z^{n}$ an entire function of order $<\frac{1}{2} . \Delta\left(E_{F}(x)\right)$ is a distribution concentrated at $x=0$. This corresponds to the facts that the $T$-product is determined by $E^{(+)}(x)$ except at the origin.

We show now that a particular choice of $E_{F}$ can be made which has no real singularities of the type $\square^{n} \delta^{(4)}(x)(n=0,1,2, \ldots)$. We construct this "minimal" superpropagator as a limiting value of an analytic function, 
using an approach which is well-known in the case of the free-field propagator $\Delta_{F}$. Let

$$
f(z)=e^{\frac{\lambda m}{\sqrt{-z}} K_{1}(m \sqrt{-z})}-1
$$

with $\lambda=f^{2} / 4 \pi^{2} . f(z)$ is regular unless $z$ is real and positive. As usual [9], [8] the Wightman distribution is given by the boundary value

$$
i E^{(+)}(x)=f\left(\left(x_{0}-i 0\right)^{2}-\boldsymbol{x}^{2}\right)
$$

Since $E_{F}(x)=E^{(+)}(x)$ if $x_{0}>0$ and $E_{F}(x)=E_{F}(-x)$, a permissible choice for the time-ordered distribution is

$$
i E_{F}(x)=f\left(x_{0}^{2}-x^{2}-i 0\right)
$$

provided we can give meaning to the right-hand side as a distribution. To this end we consider

$$
F(z)=\frac{1}{\sqrt{-z}} f(z)
$$

This is convenient since the discontinuity of $F(z)$ for $z=\xi>0$ is welldefined:

where

$$
\begin{aligned}
F(\xi+i \delta)-F(\xi-i \delta)= & \frac{i}{\sqrt{\xi}}(f(\xi+i \delta)+f(\xi-i \delta)) \\
& \underset{(\delta \rightarrow 0)}{\longrightarrow} \frac{i}{\sqrt{\xi}} f^{(1)}(\xi),
\end{aligned}
$$

$$
\begin{aligned}
f^{(1)}\left(x^{2}\right) & =i\left[E^{(+)}(x)+E^{(+)}(-x)\right] \\
& =2\left\{e^{\frac{\pi}{2} \frac{\lambda m}{\sqrt{x^{2}}} Y_{1}\left(m \sqrt{x^{2}}\right)} \cos \left[\frac{\pi}{2} \frac{\lambda m}{\sqrt{x^{2}}} J_{1}\left(m \sqrt{x^{2}}\right)\right]-1\right\} .
\end{aligned}
$$

Now

$$
F(z)=\frac{1}{2 \pi i} \int_{C} \frac{d \zeta F(\zeta)}{\zeta-z}
$$

and we split the contour into two parts: $C=C_{1}+C_{2}$
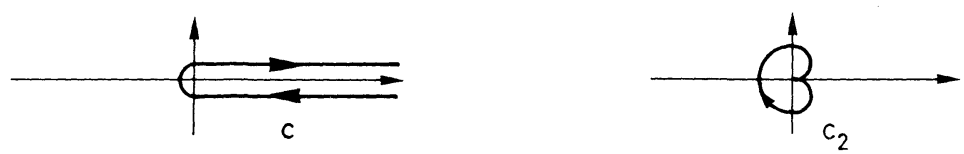
with $C_{2}$ circling the origin where $F(z)$ has an essential singularity. Then $f=f_{1}+f_{2}$,

$$
f_{i}(z)=\frac{\sqrt{-z}}{2 \pi i} \int_{C_{i}} \frac{d \zeta F(\zeta)}{\zeta-z}
$$

with $f_{1}(z)$ non-singular at $z=0$. This follows by shrinking $C_{1}$ to the positive real axis:

$$
f_{1}(z)=\frac{\sqrt{-z}}{2 \pi} \int_{0}^{\infty} \frac{d \xi}{\sqrt{\xi}} \frac{f^{(1)}(\xi)}{\xi-z}
$$

and noting that $f^{(1)}(0)=\lim _{\xi \rightarrow 0} f^{(1)}(\xi)$ is finite. Hence $f_{1}(z)$ has a welldefined limit for $z \rightarrow x^{2}-i 0$.

The singular term $f_{2}$ can be treated as follows:

$$
f_{2}(z)=\sum_{n=0}^{\infty} \frac{c_{n}}{(-z)^{n+\frac{1}{2}}}
$$

with

$$
\begin{aligned}
& c_{n}=\frac{1}{2 \pi i} \int_{C_{2}} d \zeta(-\zeta)^{n-\frac{1}{2}} f(\zeta) \\
&\left|c_{n}\right| \leqq \frac{(\text { const })^{n}}{\Gamma\left(n+\frac{3}{2}\right)}
\end{aligned}
$$

The inequality follows from the behaviour of $f(\zeta)$ in the neighborhood of $\zeta=0$. Therefore the limiting value of $f_{2}(z)$ is given by

$$
f_{2}\left(x^{2}-i 0\right)=\sum_{n=0}^{\infty} \frac{c_{n}}{\left(-x^{2}+i 0\right)^{n+\frac{1}{2}}}
$$

as a series of distributions on $\mathscr{S}\left(R^{4}\right)$, extensively studied by Gelfand and Schilow [10] which due to (14) converges to a distribution on $\mathscr{C}\left(R^{4}\right)$. This completes the definition of $f\left(x^{2}-i 0\right)$ and of $E_{F}(x)$ by means of Eq. (7). The possibility of defining $\exp \left(i f^{2} \Delta_{F}\right)$ as a limiting value of an analytic function distinguishes this expression from $\Delta_{F}^{n}$ which occurs in polynomial interactions. In the latter case, due to the singularities of $\left(x^{2}-i 0\right)^{\lambda}$ for $\lambda=-2,-3, \ldots$ the limit has no well-defined meaning.

We note the following properties of the Fourier-transform

$$
\tilde{E}_{F}(k)=\int d^{4} x \mathrm{e}^{i k x} E_{F}(x) .
$$


For the singular part we obtain $[10]$

$$
\tilde{E}_{F_{2}}(k)=8 \pi^{3} \sum_{n=0}^{\infty} \frac{(-1)^{n} c_{n}}{2^{2 n}} \frac{\left(-k^{2}-i 0\right)^{n-\frac{3}{2}}}{\Gamma\left(n-\frac{1}{2}\right) \Gamma\left(n+\frac{1}{2}\right)} .
$$

This is imaginary for $k^{2}>0$.

For the real part of $\tilde{E}_{F_{1}}(k)$ we have from (12)

$$
\operatorname{Re} \tilde{E}_{F_{1}}(k)=\frac{1}{2 \pi} \int_{0}^{\infty} d \xi \frac{f^{(1)}(\xi)}{\sqrt{\xi}} \int d^{4} x e^{i k x} \Theta\left(x^{2}\right) \sqrt{x^{2}} P \frac{1}{\xi-x^{2}} .
$$

The Fourier-integral is easily calculated and gives for $k^{2}>0$, where $\tilde{E}_{F_{2}}$ does not contribute,

$$
\operatorname{Re} \tilde{E}_{F}(k)_{\left(k^{2}>0\right)}=-\pi^{2} \int_{0}^{\infty} d \xi \sqrt{\xi} f^{(1)}(\xi) \frac{J_{1}\left(\sqrt{\xi} \sqrt{k^{2}}\right)}{\sqrt{k^{2}}} .
$$

For $m=0$ this definition is equivalent to the one proposed by Volkov [2] and Filippov [3].

(18) exhibits $\operatorname{Re} E_{F}(k)$ for $k^{2}>0$ as the Hankel-transform of an infinitely differentiable function which satisfies appropriate conditions at infinity. Therefore $\operatorname{Re} E_{F}(k)$ decreases strongly for $k^{2} \rightarrow+\infty$. Our definition is in fact the only one with this property. Any other choice differs from it by

$$
\Delta\left(\tilde{E}_{F}(k)\right)=\sum_{n=0}^{\infty} a_{n}\left(-k^{2}\right)^{n}
$$

which, being an entire function of order $<\frac{1}{2}$, does not vanish in any direction for $k^{2} \rightarrow \infty$. The corresponding property in coordinate space is:

$$
\int d^{3} x g(\boldsymbol{x}) \operatorname{Re} E_{F}(x)=\frac{1}{2 \pi} \int d k_{0} e^{-i k_{0} x_{0}} \int d^{3} k \operatorname{Re} \tilde{E}(k) \tilde{g}(\boldsymbol{k})
$$

with $\tilde{g}(k) \in \mathscr{L}\left(R^{3}\right)$, is an infinitely differentiable function of $x_{0}$, including the origin. This shows the absence of singularities of the type $\square^{n} \delta^{(4)}(x)$

We conclude this section with the following remark:

For certain models it is necessary to consider in addition to $E_{F}$, the quantity which is formally obtained by replacing $f^{2} \rightarrow-f^{2}$ (or $\lambda \rightarrow-\lambda)$, i.e.

$$
i E_{F,-\lambda}(x)=e^{-i f^{2} \Delta_{F}(x)}-1 .
$$

Without going into details, we note that also in this case a "minimal" superpropagator can be defined as a boundary value. In momentum 
space, the analogue of Eq. (18) is

$\tilde{E}_{F,-\lambda}(k)_{\left(k^{2}<0\right)}=-2 \pi^{2} \int_{0}^{\infty} d \xi \sqrt{\xi}\left[e^{-\frac{\lambda m}{\sqrt{\xi}} K_{1}(m \sqrt{\xi})}-1\right] \frac{J_{1}\left(\sqrt{\xi} \sqrt{-k^{2}}\right)}{\sqrt{-k^{2}}}$

valid for $k^{2}<0[2]$.

This decreases strongly for $k^{2} \rightarrow-\infty$. We can conclude as above that $E_{F,-\lambda}(x)$ has no real $\square^{n} \delta^{(4)}(x)$ singularities. For a more detailed investigation of properties of $E_{F}$ and $E_{F,-\lambda}$ we refer to the work of Volkov [2] and of Karowski [11].

\section{The Superpropagator for a Zero-Mass Field}

For $m=0$ the function $f(z)$ is elementary:

$$
f(z)=e^{-\frac{\lambda}{z}}-1 .
$$

The decomposition into a regular and a singular part is now given by

$$
\begin{aligned}
& f_{1}(z)=\frac{1}{\sqrt{\pi}} \Psi\left(\frac{1}{2}, \frac{1}{2} ;-\frac{\lambda}{z}\right)-1 \\
& f_{2}(z)=\sum_{n=0}^{\infty} \frac{\lambda^{n+\frac{1}{2}}}{(-z)^{n+\frac{1}{2}}} \frac{1}{\Gamma\left(n+\frac{3}{2}\right)}
\end{aligned}
$$

where $\Psi$ is a confluent hypergeometric function in the notation of Erdélyi [12]. The behaviour of $f_{1}(z)$ for $z \rightarrow 0$ follows from

$$
\Psi\left(\frac{1}{2}, \frac{1}{2} ;-\frac{\lambda}{z}\right) \underset{(z \rightarrow 0)}{\longrightarrow} \sqrt{\frac{-z}{\lambda}} .
$$

Actually, there exists an instructive different approach towards the construction of a least singular massless superpropagator $E_{F}=1 / 2\left(\bar{E}-i E^{(1)}\right)$. This is why we shall treat the zero-mass case in more detail.

The term $E^{(1)}$ being the sum of two Wightman functions is well defined. It is the term $\vec{E}(x)$ which, as product of the vacuum expectation value of the commutator $E(x)=E^{(+)}(x)-E^{(+)}(-x)$ with the antisymmetric step function $\varepsilon\left(x_{0}\right)$, needs precise definition. We want to show that a particular choice of $\bar{E}(x)$ is already suggested by the behaviour of $E(x)$ near $x=0$.

We integrate $E(x)$ with a test function $g(x) \in \mathscr{C}\left(R^{4}\right)$ [8].

$$
E(g)=\int d^{4} x g(x) E(x) .
$$


In any given frame of reference, due to the rotational invariance of $E(x)$, $g(x)$ is involved only via its average over the spatial angles:

$$
G\left(x_{0}, s\right)=\int d \Omega^{(3)} g\left(x_{0}, \boldsymbol{x}\right) /|\boldsymbol{x}|^{2}=s .
$$

We expand $E(g)$ in powers of $\lambda$ and note that the support of each term of this expansion is concentrated on the surface of the light-cone $x_{0}^{2}=s$. Next, we reexpress by means of partial integration all time derivatives of $G\left(x_{0}, s\right)$ appearing in $E(g)$ except those that enter into the boundary terms by appropriate derivatives of $G$ with respect to s. Finally, gathering all terms that involve the same derivative of $G\left(x_{0}, s\right)$ with respect to $s$, we find

$$
\begin{aligned}
E(g)= & \left(\int_{0}^{\infty} d x_{0} \sum_{l=0}^{\infty} \frac{\partial^{l}}{\partial s^{l}} G\left(x_{0}, s\right)_{s=x_{0}^{2}} \cdot A_{l}\left(x_{0}^{2}\right)+R(g)\right) \\
& -\left(\int_{-\infty}^{0} d x_{0} \sum_{l=0}^{\infty} \frac{\partial^{l}}{\partial s^{l}} G\left(x_{0}, s\right)_{s=x_{0}^{2}} \cdot A_{l}\left(x_{0}^{2}\right)-R(g)\right) \\
= & E_{R}(g)-E_{A}(g) .
\end{aligned}
$$

We have put

$$
\begin{aligned}
R(g)= & -\frac{\pi}{2} \lambda^{\frac{5}{2}} \sum_{l=0}^{\infty} \sum_{k=0}^{\infty} \frac{(-\lambda)^{l} l ! \Gamma\left(k+\frac{3}{2}\right) \cdot \lambda^{k}}{(2 l+1) ! \Gamma\left(l+k+\frac{5}{2}\right) \Gamma\left(l+k+\frac{7}{2}\right) \cdot k !} \\
& \cdot \frac{\partial^{2 l+1}}{\partial x_{0}^{2 l+1}} \frac{\partial^{k}}{\partial s^{k}} G(0,0) .
\end{aligned}
$$

$A_{l}\left(x_{0}^{2}\right)$ is a hypergeometric function which is infinitely differentiable and has the following asymptotic expansion for small $x_{0}$

$$
A_{l}\left(x_{0}^{2}\right) \sim \frac{\lambda^{\frac{3}{2}}}{2 \sqrt{\pi}} \frac{(-\lambda)^{l}}{l !} \sum_{n=0}^{\infty}\left(\frac{x_{0}^{2}}{\lambda}\right)^{n} \frac{\Gamma\left(n-\frac{1}{2}\right) \Gamma\left(n-l-\frac{3}{2}\right)}{n !} .
$$

The indicated splitting into a contribution from $x_{0} \geqq 0$ and $x_{0} \leqq 0$ is Lorentz-invariant though not unique. The point $x_{0}=0$ is distinguished in two ways: first, as the end of the domains of integration; second as the support of $R(g)$.

After having smeared $E(x)$ in the space arguments it is infinitely differentiable in the remaining time argument away from the origin. There is now one Lorentz-invariant choice of the product of $E(x)$ with the step function $\varepsilon\left(x_{0}\right)$, namely

$$
\bar{E}(g)=E_{R}(g)+E_{A}(g)=\int_{-\infty}^{+\infty} d x_{0} \sum_{l=0}^{\infty} \frac{\partial^{l}}{\partial s^{l}} G\left(x_{0}, s\right)_{s=x_{0}^{2}} \cdot A_{l}\left(x_{0}^{2}\right)
$$


that is moreover infinitely differentiable in the time argument at the origin after the partial smearing. A different choice would introduce singular contributions at the point $x_{0}=0$ which is not singled out in the above expression. (25) coincides with our earlier definition.

One may describe the procedure slightly differently:

$\bar{E}(x)$ is defined uniquely on the space of test functions contained in $\mathscr{C}\left(R^{4}\right)$ which vanish together with all their derivatives at the point $x=0$. Define $\bar{E}(g)$ on all $g \in \mathscr{C}\left(R^{4}\right)$ as the limit $t \rightarrow 0$ of $\bar{E}\left(g \cdot \psi_{t}\right)$ where

$$
\begin{gathered}
\psi_{t}(x) \in \mathscr{C}_{M}\left(R^{4}\right) \text { is a multiplier of } \mathscr{C}\left(R^{4}\right), \\
0 \leqq \psi_{t}(x) \leqq 1 \\
\psi_{t}(x)=1 \text { for } x_{0}^{2}+x^{2}>t, \\
\frac{\partial^{k_{0}}}{\partial x_{0}^{k_{0}}} \cdots \frac{\partial^{k_{3}}}{\partial x_{3}^{k_{3}}} \psi_{t}(x)_{x=0}=0 \text { for all non-negative integers } k_{0}, \ldots, k_{3},
\end{gathered}
$$

$\psi_{t}(x)$ is uniformly bounded in $t$ in an appropriate topology that involves the values of $\psi_{t}(x)$ and of its spatial derivatives (but not its time derivatives) on the surface of the light-cone only.

The limit exists, is independent of the special choice of the sequence $\psi_{t}$, and coincides with (25). Clearly, $\bar{E}(x)$ does not have singularities of the type $\square^{n} \delta^{(4)}(x), n=0,1,2, \ldots$.

\section{Second-Order Terms in a Model with Exponential Coupling}

The minimal superpropagator we have defined is characterized by the absence of real singularities $\square^{n} \delta^{(4)}(x)$. We denote it for the moment by $E_{F}^{\{0\}}$. The general superpropagator can then be labeled by a sequence of real numbers $a_{n}$

$$
E_{F}^{\left\{a_{n}\right\}}(x)=E_{F}^{\{0\}}(x)+\sum_{n=0}^{\infty} a_{n} \square^{n} \delta^{(4)}(x) .
$$

It is well known [13] that the singularities in question play a special role. They are related to the addition of higher order terms to an interaction Lagrangian which is given to first order in some coupling constant $G$. To illustrate the significance of our choice of superpropagator for second order effects we consider a simple model of an exponentially self-coupled scalar field.

Let the first order interaction Lagrangian be

$$
\mathscr{L}_{1}=G:\left(e^{f \phi_{0}}-1-f \phi_{0}-\frac{f^{2} \phi_{0}^{2}}{2}\right):
$$

We take $\phi_{0}$ as the incoming field to the interacting field

$$
\phi(x)=\phi_{0}(x)-\int d^{4} x^{\prime} \Delta_{R}\left(x x^{\prime}\right) j\left(x^{\prime}\right)
$$


and expand

$$
\phi=\phi_{0}+\sum_{n=1}^{\infty} G^{n} \phi_{n} ; \quad j=\sum_{n=1}^{\infty} G^{n} j_{n} .
$$

The first order term is given by $\mathscr{L}_{1}$ as

$$
j_{1}=f:\left(e^{f \phi_{0}}-1-f \phi_{0}\right): .
$$

Due to the singular structure of the free-field commutator, the second order field generated by $\mathscr{L}_{1}$ is not uniquely determined. Using standard methods we find

$$
\begin{gathered}
j_{2}(x)=-f \int d^{4} \xi\left\{E_{R}^{\prime}(x-\xi):\left[\left(e^{f \phi_{0}(x)}-1\right)\left(e^{f \phi_{0}(\xi)}-1\right)+e^{f \phi_{0}(\xi)}-1\right.\right. \\
\left.\left.-f \phi_{0}(\xi)\right]:+f^{2} \Delta_{R}(x-\xi):\left(e^{f \phi_{0}(x)}-1\right)\left(e^{f \phi_{0}(\xi)}-1-f \phi_{0}(\xi)\right):\right\} \\
E_{R}(x)=E_{F}(x)-E^{(+)}(-x)
\end{gathered}
$$

is the retarded superpropagator. In

$$
E_{R}^{\prime}(x)=E_{R}(x)-f^{2} \Delta_{R}(x)
$$

the one-particle contribution has been subtracted.

Of course, as shown by (31), the ambiguities of the second order field and of the superpropagator have the same origin. We can label the various fields as $\phi_{2}^{\left\{a_{n}\right\}}$ which is defined as the second order field constructed with $E_{R}^{\left\{a_{n}\right\}}$. We call $\phi_{2}^{\{0\}}$ the minimal second order field associated with the Lagrangian (27). Then

$$
\begin{aligned}
j_{2}^{\left\{a_{n}\right\}}= & j_{2}^{\{0\}}-f \sum_{n=0}^{\infty} a_{n}:\left\{\left(e^{f \phi_{0}}-1\right) \square^{n}\left(e^{f \phi_{0}}-1\right)\right. \\
& \left.+\square^{n}\left(e^{f \phi_{0}}-1-f \phi_{0}\right)\right\}:
\end{aligned}
$$

We can argue that $j_{2}^{\{0\}}$ corresponds most closely to the original Lagrangian without the addition of finite second order terms as in (32). To support this view we note for example that all additional terms with $n \geqq 1$ contain even order time derivatives of $\phi_{0}$. It can be shown that the matrix elements of $j_{2}^{\{0\}}$ between suitable incoming states do not involve such derivatives [14]. The weakness of such arguments lies in their appeal to minimal singularity in models which remain nevertheless very singular. In perturbation theory, to which the whole discussion is restricted, they lead necessarily to cross sections which rise strongly with energy. Exact solutions, if they exist, have a reasonable high-energy behaviour [15]. In view of this situation the significance of perturbation theory arguments is hard to assess. Hopefully, the relation between "absence of $\delta$-singularities" and absence of derivative coupling terms holds more generally, and our construction corresponds to a simple dynamical structure. 


\section{References}

1. Okubo, S.: Prog. Theor. Phys. 11, 80 (1954).

2. Volkov, M. K.: Commun. Math. Phys. 7, 289 (1968) and 15, 69 (1969).

3. Filippov, A. T.: Partial symmetry, equivalence theorems and calculation of higher orders in non-renormalizable field theory. DUBNA preprint (1968).

4. Efimov, G. V.: Review of methods in non-linear quantum field theory. CERN preprint (1969) and references quoted there.

5. Delbourgo, R., Salam, A., Strathdee, J.: Phys. Rev. 187, 1999 (1969); Salam, A.: Nonpolynomial Lagrangian theories, Trieste preprint (1970), to appear in the Proceedings of the Coral Gables Conference 1970.

6. Lee, B. W., Zumino, B.: Nuclear Phys. B 13, 671 (1969).

7. Keck, B. W., Taylor, J. G.: Renormalization of non-polynomial Lagrangians. University of Southampton preprint (1970).

8. Jaffe, A.: Phys. Rev. Letters 17, 661 (1966), Phys. Rev. 158, 1454 (1967).

9. Wightman, A. S.: Phys. Rev. 101, 860 (1956).

10. Gelfand, I. M., Schilow, G. E.: Verallgemeinerte Funktionen I. Berlın: VEB Deutscher Verlag der Wissenschaften 1960.

11. Karowski, M.: To be published.

12. Erdélyi, A.: Higher transcendental functions, Vol. I. New York: McGraw-Hill, Inc. 1953.

13. Bogoliubov, N. N., Shirkov, D. V.: Introduction to the theory of quantized fields. New York: Interscience Publ., Inc. 1959

14. Lehmann, H., Pohlmeyer, K.: To appear in the Proceedings of the Second International Symposium on non-local Quantum Field Theory, Azau, USSR (1970).

15. Epstein, H., Glaser, V., Martin, A.: Commun. Math. Phys. 13, 257 (1969).

H. Lehmann u. K. Pohlmeyer

II. Institut für theoretısche Physık

der Universität

2000 Hamburg 50, Luruper Chaussee 149 